\title{
Priority oral health research identification for clinical decision-making
}

Authors

Helen Worthington, Jan Clarkson, Jo Weldon

Institution

Cochrane Oral Health Group, School of Dentistry, The University of Manchester.

Key words

systematic reviews, evidence-based medicine, dentistry/healthcare, clinical studies/trials, comparative effectiveness research (CER)

\section{Abstract}

The Cochrane Library is a core resource for clinical decision-making globally, by clinicians, guideline developers, healthcare providers and patients.

The publication of Cochrane Library systematic reviews concerning oral health conditions has grown exponentially to over 215 individual titles (as of 20 June 2015) during the past 20 years.

Consequently, maintaining updates of the most clinically important reviews to provide up-to-date and accurate sources of evidence for decision-making has become a pressing concern for the editorial group behind their production, Cochrane Oral Health Group.

To identify priority research required by oral health decision-makers, the Cochrane OHG embarked on a consultation process across eight defined areas of dentistry (periodontology, operative (including endodontics) and prosthodontics, paediatric dentistry, dental public health, oral and maxillofacial surgery, oral medicine, orthodontics, cleft lip and/or palate) with existing authors (by email), with members of the public (by online survey), and established internationally clinically expert panels for each area of defined area of dentistry to discuss and ratify (by teleconference) a core portfolio of priority evidence to be produced and maintained on the Cochrane Library.

The resulting portfolio of priority research encompasses 81 existing titles to be maintained, and an additional 15 new systematic reviews to be developed by the Cochrane OHG in due course.

The Cochrane OHG has actively responded to the outcomes of this prioritisation process by allocating resources to primarily supporting the maintenance of identified priority evidence for the Cochrane Library.

\section{Background}

The ability to easily access accurate, up-to-date and clinically relevant evidence for oral health prevention and treatment has become increasingly important to an array of stakeholders; whether they are clinicians, guideline developers, healthcare providers or patients.

The Cochrane Library is a global leader in publishing methodologically-sound systematic reviews to provide accurate synopses of the best evidence of oral health care interventions, and currently occupies $10^{\text {th }}$ position in the world Impact Factor (IF) rankings for Medicine, General and Internal journals (at 5.9). Furthermore, the Cochrane OHG's individualised (IF) ranking of its 215 publications (159 reviews and 56 protocols) hosted on the Cochrane Library places its output in $3^{\text {rd }}$ position in the world rankings for Dentistry, Oral Surgery and Medicine journals (at 4.00; behind Dental Materials [4.16], and JDR [4.14]), showing the impact of the Cochrane OHG's reviews on clinical decisionmaking around the globe.

Despite demonstrating significant impact upon publication, it is important to sustain regular updates of each systematic review and this requires additional investment (to the existing core infrastructure funding provided by National Institute for Health Research (NIHR), UK). The required funding has now been sourced from the Global Alliance (GA), an international research partnership of dental professional groups and independent donors established in 2010 to assist Cochrane OHG in providing clinical research direction and securing the necessary capacity funding. (See acknowledgements for details of all current partners).

Following the acquisition of methodological support required to produce and publish the Cochrane Oral Health Group's reviews, the Group undertook a global consultation process with international clinical experts, the general public and existing authors to identify a core portfolio of new and existing priority research titles required by a range of stakeholders for clinical decision-making.

\section{Methods}

In 2011 and 2013, two prioritisation exercises were conducted in orthodontics and paediatric dentistry respectively. The methods developed for these exercises were then used in the later ones (in periodontology, operative (including endodontic) and prosthodontic dentistry, dental public health, oral and maxillofacial surgery, oral medicine, cleft lip and/or palate), which took place in 2014. 


PERIODONTOLOGY
1. Primary prevention
(recall intervals; scale \& polish; toothbrush types)
2. Maintenance
(dentition; OHI adherence; interdental cleaning)
3. Other priorities
(OHI for VAP prevention; perio treatment for glycaemic control;
perio treatment for CVD management)
ORAL MEDICINE
1. Oral cancer
2. Oral conditions
(Screening; diagnostic tests; clinical assessment)
(TMD; OLP; leukoplakia; denture stomatitis)

CLEFT LIP +/- PALATE
management; prevention; SLP intervention

OPERATIVE \& PROSTHODONTIC DENTISTRY
1. Operative/endodontic treatment
(caries management; fillings; restoration maintenance; root-filled
teeth restoration; treatment duration; retreatment options)
2. Prosthodontic treatment
(implant timing after extraction; implant load timing; partially-absent
dentition; prostheses for edentulous patients)

\section{ORTHODONTICS}

Class II \& III malocclusion treatment; crowded teeth; anchorage reinforcement; position retention procedures; unerupted palatally displaced permanent canine extraction; posterior crossbites; initial archwire alignment; non-surgical tooth movement acceleration; deep bite treatment
DENTAL PUBLIC HEALTH

oral health promotion (i. community; ii. institutionalised care); water fluoridation; oral cancer screening; OHI for VAP prevention; sealant delivery/promotion programmes (i. schools; ii. communities); school dental screening; ECC prevention; elderly caries prevention

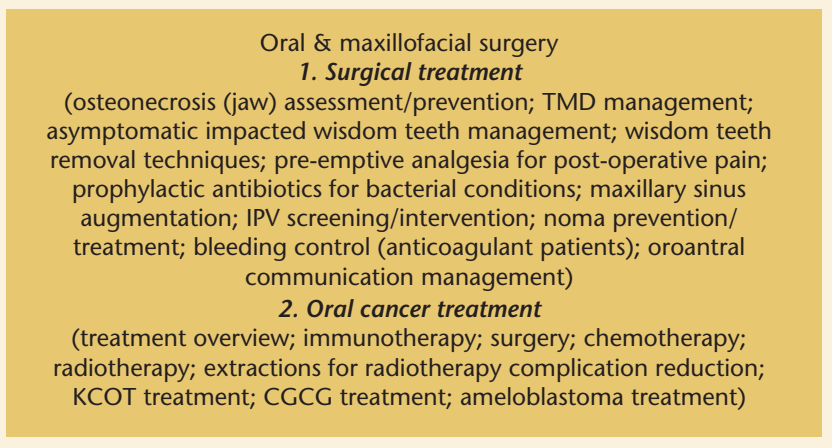

PAEDIATRICS
1. Topical fluoride
(varnish; gel; mouthrinse; toothpaste; overview; combination;
comparison; toothpaste concentration)
2. Sealants
(caries prevention; sealant vs. varnish)
3. Other priorities
(ART efficacy; pulp treatment; non-fluoride topical remineralisation;
minimally-invasive caries management; preoperative analgesics;
fillings; operative caries management; preformed metal crowns;
non-pharmalogical anxiety management)

Figure 1. Spread of reported oral health concerns to inform decision-making

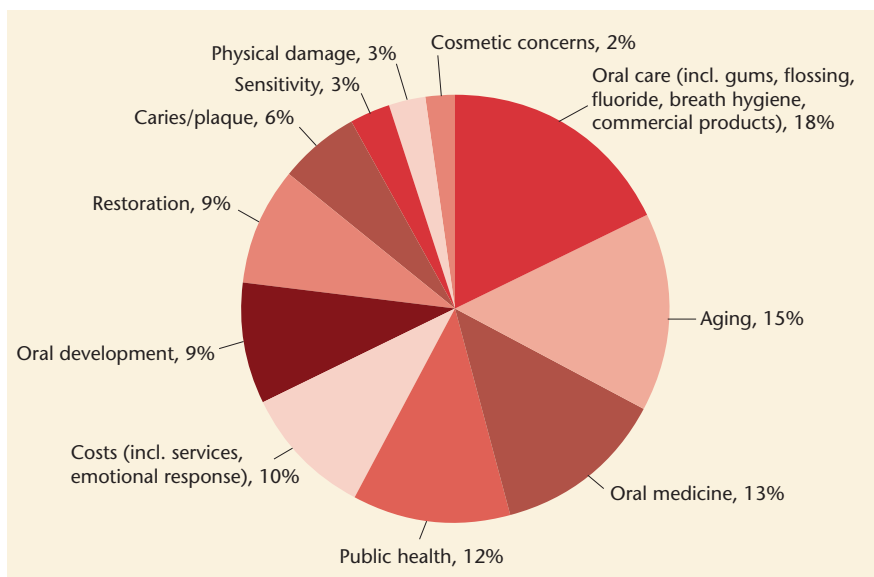

Figure 2. Identified research priorities for oral health

Authors of all relevant existing Cochrane Reviews from each of the eight defined areas of dentistry were asked to provide initial 'top 10 ' rankings of what they considered to be the most clinically important existing titles (using a spreadsheet of reviews, protocols and registered titles), and to identify other important review topics to consider for registration.

An international expert panel (EP) was assembled for each dental area by consulting members of the GA, Cochrane Editors, known clinical and research experts and high-impact journal editorial board members, and they were invited to take part in the prioritisation exercise.
Public opinion was elicited by an online open consultation survey, which asked participants to disclose their oral-health concerns, and to pose three oral-health related questions to which they would like answers. Eighty-one people responded during a two-week period, from 14 countries across five continents.

Each EP (ranging from 11 to 22 members) was sent a summary of the 15 highest-ranked relevant titles pooled from the initial authors' results, new title suggestions for their area, and the full list of existing oral health titles registered on the Cochrane Library for their consideration. They were also asked to consider whether any important gaps in clinical evidence were missing from the existing research portfolio.

An EP teleconference, chaired by the Cochrane OHG's two coordinating editors (Jan Clarkson and Helen Worthington), was conducted for each area of dentistry and provoked much debate into what the important reviews in each area were, and which, if any, new research titles needed to be developed.

\section{Results}

The public consultation survey indicated that respondents were most frequently concerned about oral health maintenance, prevention of gum disease and decay, and that the priority groups were the elderly and children. Figure 1 provides an indication of the spread of reported consumer concerns. Relevant concerns were outlined at the start of each EP discussion.

After consideration of global oral health needs, cultural differences, economic drivers and services delivery, the EP teleconferences 
resulted in revised rankings from those initially generated. The number of priority reviews was originally intended to be limited to around ten titles for each area (simply to result in a manageable portfolio), but this was flexible and ultimately decisions were guided by each EP.

Eighty-one existing titles were identified as being priority research for maintenance, and the scope of some titles has expanded to provide more clinically useful evidence for the profession, with a few existing titles being merged to rationalise the evidence, and in some areas the development of overviews was considered. Additionally, 15 new priority titles have been proposed for development by the Cochrane OHG, resource permitting.

The issue of professional groups independently undertaking systematic reviews also generated discussion on duplication of effort whilst recognising the quality and independence of Cochrane reviews. Results of the prioritisation process are available on the Cochrane OHG website at ohg.cochrane.org/priority-reviews.

\section{Conclusion}

This prioritisation and rationalisation process has been an enormous achievement in establishing a core portfolio of clinically important evidence for oral health, and now allows the Cochrane OHG to strategically direct evidence publication on the Cochrane Library, in accordance with key stakeholders' needs for clinical practice.

The Cochrane OHG is tremendously grateful to the consideration and time provided by the EP members, existing review authors and survey respondents. The core portfolio will be reassessed for clinical relevance in two years.

\section{Acknowledgements}

Sources of support: All prioritisation activities were funded by the Global Alliance.

To date, GA partners include: British Orthodontic Society, UK; Royal College of Surgeons of Edinburgh, UK; New York University, USA; British Society of Paediatric Dentistry, UK; National Center for Dental Hygiene Research \& Practice, USA; Canadian Dental Hygienists Association, Canada; British Society of Periodontology, UK; British Association of Oral Surgeons, UK; Mayo Clinic, USA; British Association for the Study of Community Dentistry, UK.

Conflicts of interest: The authors declare no conflicts of interest exist.

Evidence-Based Dentistry (2015) 16, 69-71. doi:10.1038/sj.ebd.6401110 